\title{
A CASE REPORT OF CANINE EHRLICHIA INFECTION IN A LABRADOR DOG AND ITS THERAPEUTIC MANAGEMENT
}

\author{
D. Barman, B. C. Baishya, D. Sarma, A. Phukan and T. C. Dutta \\ Department of Veterinary Clinical medicine, Ethics \& Jurisprudence, College of Veterinary Science, AAU, \\ Khanapara, Guwahati - 781022, Assam, India
}

\begin{abstract}
Ehrlichiosis is an important protozoan disease in canine caused by an intracellular gram - negative bacteria of the genus Ehrlichia, under the family Anaplasmataceae. The important species under the genus Ehrlichia are E. canis, E. ewingii and E. chaffeensis. Another two bacteria within the family Anaplasmataceae are Anaplasma platys (Syn: E. platys) and A. phagocytophilum; on the other hand, E. platys and A. phagocytophilum are synonymous. Prevalence of ehrlichiosis remains high in north eastern region of India especially Assam but it remains undiagnosed due to lack of owner's awareness. The incidences of ehrlichiosis in dog in Guwahati caused by E. canis and E. platys are recorded which are primarily responsible for canine monocytic ehrlichiosis and canine thrombocytic as well as granulocytic ehrlichiosis, respectively. A case of ehrlichiosis in canine was diagnosed based on clinical signs, blood smear examination and polymerase chain reaction (PCR). The dog was treated with two doses of diminazine diaceturate deep intramuscularly at 48 hours interval and doxycycline tablet orally for one month besides supportive therapy rendered during the period. After a month of treatment the dog showed recovery and by two months it recovered completely. Thus it can be inferred that, doxycycline along with other supportive medication for about a month could cure a critical case of canine ehrlichiosis.
\end{abstract}

Key words: splenomegaly, hepatomegaly, anemia, ehrlichia, doxycycline

Ehrlichiosis occurrence in canine usually remains preclinical. The north eastern zone of India, particularly Assam is a reservoir of parasites including ticks and fleas. Ehrilichia is not uncommon in this region but it remains undiagnosed because of lack of awareness amongst clients. In the Teaching Veterinary Clinical Complex, College of Veterinary Science, Khanapara, Guwahati, Assam, India, several cases of ehrlichiosis caused by E. canis and E. platys were recorded. Few cases had mixed infection with Babesia gibsoni and B. canis. In Kolkata, India, 47 cases of ehrlichiosis were reported having mixed infection with babesiois $(8.1 \%)$ and hepatozoonosis $(6.38 \%)$ by Mausam Das et al. (2013). Clinical findings recorded in general were pale visible mucous membrane (Fig. 3), initial high rise of temperature, inappetence, marked weight loss, dark colour urine, hepatomegaly and splenomegaly. The case was confirmed by demonstration of the organism as E. platys on blood smear by laboratory procedure, although PCR is considered to be the most diagnostic and confirmatory test. The incubation period of canine ehrlichiosis ranges from 8 to 20 days, after which the clinical entity reveals 3 phases: acute, subclinical, and chronic (Harrus et al. 1998). Once diagnosis was confirmed to be ehrlichiosis, a prolong therapeutic management was required to recover the ailing $\mathrm{dog}$.

\section{CASE HISTORY AND OBSERVATION}

An eight and half year old, male, Labrador dog (19 kg body wt) was presented at the Teaching Veterinary Clinical Complex, College of Veterinary Science, Guwahati, Assam, India. It was having history of inappetence, fever, weakness, anemia, scanty feces, hemoglobinuria, shrunken eye ball with mild corneal opacity and reluctant to walk and that might be due to pain in the joints. On physical inspection capillary refill time increased to 3 seconds, penile mucous membrane was pale (Fig. 3), second degree of dehydration and on palpation splenomegaly and partial hepatomegaly can be observed (Parmar et al., 2013). Sonographic investigation of the abdomen revealed rounding of the borders of liver and expanded spleen. Due to this enlargement, there was a pressure build on diaphragm towards cranial aspect leading to partial dyspnoea. Blood sample was collected in EDTA vial for routine examination. Peripheral blood smear was made from the animal and subjected to direct microscopic examination using Giemsa stain for hemoprotozoan parasite investigation. The routine examination of blood revealed hemoglobin concentration $5.1 \mathrm{~g} \%$, RBC $3.13 \mathrm{~m} / \mathrm{mm}^{3}$, thrombocyte count was $21 \mathrm{~m} / \mathrm{mm}^{3}$ (table $1)$.

*Corresponding e-mail address: ditulbarman@ rediffmail.com 


\section{Barman and others}

Microscopic examination of stained blood smear revealed E. platys, intracytoplasmically in the thrombocytes (Fig. 1). Further, the diagnosis of E. platys was confirmed through PCR. Thus on the basis of laboratory and clinical examination ehrlichiosis was confirmed. Similar findings were also observed by Waner et al. (1999). Further blood serum was collected for investigation of kidney and liver function tests. The blood serum creatinine level was $1.7 \mathrm{mg} / \mathrm{dl}$ and BUN was $210 \mathrm{mg} / \mathrm{dl}$. The AST and ALT levels were $178 \mathrm{u} / \mathrm{l}$ and $124 \mathrm{u} / \mathrm{l}$, respectively, revealing that the liver was in stressful condition. The rectal temperature was $104^{0} \mathrm{~F}$ initially but subsided after commencement of the treatment and thereafter came down to normal.

Table 1. Values of the hematological parameters of the dog infected by Ehrlichiosis

\begin{tabular}{|lll|}
\hline Parameters & Values & Reference \\
\hline $\mathrm{RBC}\left(\mathrm{m} / \mathrm{mm}^{3}\right)$ & $3.13 \pm 1.76$ & $5.5-8.5$ \\
$\mathrm{Hb}(\mathrm{g} / \mathrm{dL})$ & $5.10 \pm 2.12$ & $12.0-18.0$ \\
$\mathrm{PCV}(\%)$ & $22.34 \pm 11.18$ & $37-55$ \\
$\mathrm{WBC}\left(\mathrm{m} / \mathrm{mm}^{3}\right)$ & $3.38 \pm 1.32$ & $6-17$ \\
Neutrophil $\left(\mathrm{m} / \mathrm{mm}^{3}\right)$ & $6.30 \pm 1.08$ & $3-11.5$ \\
Eosinophil $\left(\mathrm{m} / \mathrm{mm}^{3}\right)$ & $0.39 \pm 0.27$ & $0.1-1.25$ \\
Basophil $\left(\mathrm{m} / \mathrm{mm}^{3}\right)$ & $0.0 \pm 0.0$ & 0 \\
Lymphocyte $\left(\mathrm{m}^{3} / \mathrm{mm}^{3}\right)$ & $1.09 \pm 0.67$ & $1-4.8$ \\
Monocyte $\left(\mathrm{m} / \mathrm{mm}^{3}\right)$ & $0.15 \pm 0.08$ & $0.15-1.35$ \\
Thrombocyte $\left(\mathrm{m} / \mathrm{mm}^{3}\right)$ & $21.0 \pm 9.19$ & $200-500$ \\
\hline
\end{tabular}

\section{TREATMENT AND DISCUSSION}

Once the blood sample collected, the Labrador was treated with Fenbedazole @ $7.5 \mathrm{mg} / \mathrm{kg}$ bwt (Panacur from MSD animal health India Ltd.), Iron oral supplement (Livoferol from Provimi Animal Nutrition, India). After laboratory confirmation as ehrlichia positive, the dog was administered Doxycycline capsule (Doxyleb $100 \mathrm{mg}$ from Leben labs, India) @ $10 \mathrm{mg} / \mathrm{kg}$, PO twice daily after food for 1 month. Similar treatment was also administered by Waner et al. (1999). Moreover, Diminazine diaceturate injection, (Nilbery from Intas Pharma, India) @ $7 \mathrm{mg} / \mathrm{kg}$ was administered deep IM at 48 hours interval for 2 occasions. Other supportive treatments included, Pantoprazole (Pan 40 from Alkem, India) @ $40 \mathrm{mg} / 20 \mathrm{~kg}$ bwt was mixed with IV fluid at 12 hours interval for initial 7 days, Ondansetron (Vomikind inj. from Mankind, India) @ $1 \mathrm{mg} / \mathrm{kg}$ IM at 12 hourly for 7 days. Vitamin B complex (Conciplex from Concept Pharma, India) @ 2ml along with fluid twice daily for 10 days, Colloid therapy (Haemaccel from AHPL, India) @ $10 \mathrm{ml} / \mathrm{kg}$ bwt together with Dextrose Normal Saline (Baxter India Ltd) intermittently for 15 days (Fig. 2). Sarma et al. (2013) found Haemaccel as the effective plasma expander in treating Hepatozooan canis infection in dog. To combat hypoproteinemia, essential amino acid IV fluid supplement was administered (Astymin 3, from Allianz Biosciences (P) Ltd, India) @ $20 \mathrm{ml}$ daily with IV fluid for 7 days. Normal saline was administered during elevation phase of body temperature while it was replaced with dextrose when temperature was subsided. After three weeks the Labrador started taking small amount of liquid diet and rare or no vomition was noted. To relieve from gut irritation Sucralfate (Sucra gel from Sain pharma, India) was administered orally @ 1 teaspoon thrice daily for 20 days. Glucosamine Hydrochloride and Chondroitin Sulphate tablet (Pet Joint tablet from Provimi Animal Nutrition India Pvt Ltd) @ 1 tablet PO for 1 month showed remarkable response in relieving joint pain due to polyarthritis. The anemic condition was improved following IV iron supplementation (OVERCOM $5 \mathrm{ml}$ Ampoule from Panacea Biotec, India) @ $5 \mathrm{ml}$ slow IV along with normal saline administered on alternate day for 3 occasions. Finally, after 30 days of treatment the mucous membrane started appearing pinkish and the hemoglobin level was close to $10 \mathrm{~g} / \mathrm{dl}$. The dog showed complete recovery after one month with normal appetite, general improvement of the health with playful nature.

From the laboratory report it was found that there was reduction of Hemoglobin, RBCs, WBCs and Thrombocytes indicating anemia, leucopenia and thrombocytopenia. An immunological mechanism may be involved in the pathogenesis of the chronic form of the disease through production of antibodies and their subsequent binding on the membranes of erythrocytes and platelets which may result in destructions of these cells resulting in hemoglobinuria, anemia and thrombocytopenia (Taylor et al., 2007). Further, anemia may be attributable to destruction of RBCs and bleeding tendencies that result from thrombocytopenia and platelets 


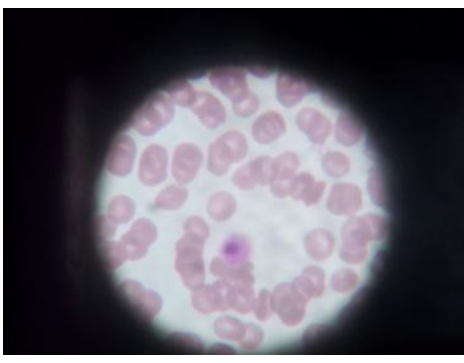

Fig. 1. Ehrlichia organism can be visualized inside the thrombocyte

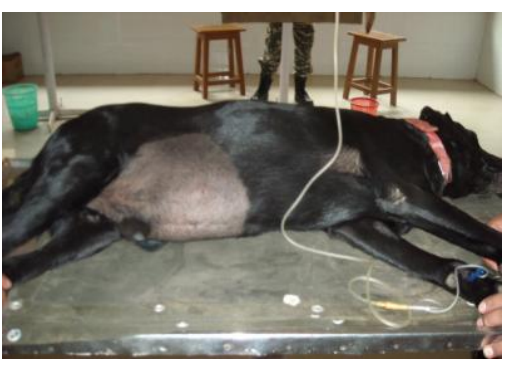

Fig. 2. Labrador was receiving fluid therapy after ultrasonography examination

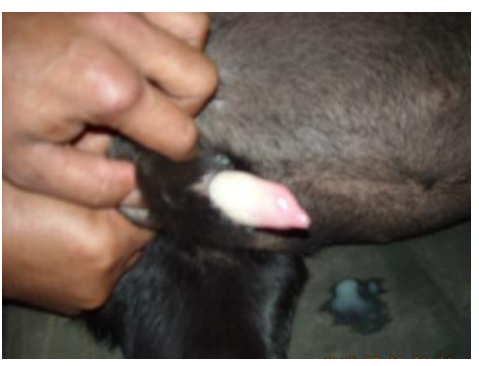

Fig. 3. The Labrador dog with pale penile mucous membrane

destruction as well as suppression of bone marrow function (Ettinger and Fieldman, 2010). The dog owners may be suggested to control the disease ehrlichiosis by controlling the tick population of Rhipicephalus sanguineous. Common acaricides such as Amitraz, Fipronil and Pyrethrins are effective and may be used as per the instruction of the manufacturer.

\section{REFERENCES}

1. Das M and Konar S (2003). Clinical and hematological study of canine Ehrlichiosis with other hemoprotozoan parasites in Kolkata, West Bengal, India. Asian Pacific Journal of Tropical Biomedicine 3(11): 913-915.

2. Ettinger SJ and Fieldman EC (2010). In. Text book of Veterinary Internal Medicine. $7^{\text {th }}$ edn, Volume I, Saunders Elsevier, p. 901-906.

3. Harrus S, Waner T, Keysary I, Aroch I, Voet H and Bark H (1998). Investigation of splenic functions in canine monocytic ehrlichiosis. Veterinary Immunology and Immunopathology 62: 15-27.

4. Parmar C, Pednekar R, Jayraw A and Gatne M (2013). Comparative diagnostic methods for canine ehrlichiosis. Turkish Journal of Veterinary and Animal Sciences 37: 282-290.

5. Sarma K, Mondal DB, Saravanan M, Kumar M and Mahendran K (2012). Haemato-biochemical changes in Hepatozoon canis infected dog before and after therapeutic management. Journal of Veterinary Parasitology 26 (1): 35-38.

6. Taylor MA, Coop RL and Wall RL ( 2007). In. Veterinary Parasitology. $3^{\text {rd }}$ edn, Blackwell Publishing, p. 420-424.

7. Waner T, Keysary A, Bark H, Sharabani E and Harrus S (1999). Canine monocytic ehrlichiosis - an overview. Israel Journal of Veterinary Medicine 54: 103-107. 